\title{
Linfedema: actualización en el diagnóstico y tratamiento quirúrgico
}

\author{
Nicolás Pereira C. ${ }^{1,2}$ y Isao Koshima ${ }^{3}$
}

\section{Lymphedema: update in diagnosis and surgical treatment}

Lymphedema is the accumulation of protein-rich fluid in the interstitium, secondary to abnormalities in the lymphatic transport system. In developed countries it is more often related to surgical treatment of cancer. The clinical diagnosis and through imaging techniques is fundamental to evaluate the functional status of the lymphatic system. The main objectives in managing lymphedema are to limit patient morbidity, improve functionality and quality of life. Surgical treatment is an option when conservative measures are no longer sufficient. There are procedures that seek to prevent the development of lymphedema, and techniques that include physiological (reconstructive) and resective procedures that are used when lymphedema is already established. Success depends on a good selection of patients and the completion of an individualized treatment. The following is a review article of the latest diagnostic strategies and update in surgical techniques with emphasis on microsurgical treatment.

Key words: lymphedema; indocyanine green lymphography; supermicrosurgery; lymphovenous anastomosis; vascularized lymph-node transfer.

\section{Resumen}

El linfedema es la acumulación de fluido rico en proteínas en el intersticio, secundario a anomalías en el sistema de transporte linfático. En países desarrollados se relaciona más frecuentemente al tratamiento quirúrgico del cáncer. El diagnóstico clínico y a través de técnicas de imágenes es fundamental para evaluar el estado funcional del sistema linfático. Los objetivos principales en el manejo del linfedema son limitar la morbilidad del paciente, mejorar la funcionalidad y la calidad de vida. El tratamiento quirúrgico es una alternativa cuando las medidas conservadoras ya no son suficientes. Existen procedimientos que buscan prevenir el desarrollo del linfedema y técnicas que incluye procedimientos fisiológicos (reconstructivos) y resectivos que se utilizan cuando el linfedema ya está establecido. El éxito depende de una buena elección de los pacientes y la realización de un tratamiento individualizado. A continuación se presenta una revisión en cuanto a las últimas estrategias diagnósticas y actualización en las técnicas quirúrgicas con énfasis en el tratamiento microquirúrgico.

Palabras clave: linfedema; linfografía con verde indocianina; supermicrocirugía; anastomosis linfáticovenosa; transferencia de linfonodos vascularizada.

\section{Introducción}

El linfedema es la acumulación de fluido rico en proteínas en el intersticio, secundario a anomalías en el sistema de transporte linfático. Independiente de su etiología, se puede manifestar como edema blando con fóvea que puede progresar a un edema sin fóvea y crecimiento irreversible de la extremidad, llevando a una fibrosis progresiva, obstrucción de los vasos linfáticos y celulitis a repetición ${ }^{1}(\mathrm{Fi}$ gura 1).
El linfedema primario se debe a anomalías en el desarrollo del sistema linfático, mientras que el secundario es consecuencia del daño de vasos linfáticos debido al trauma, cáncer, cirugía o infección. Las zonas más comprometidas son la extremidad inferior $(90 \%)$, extremidad superior $(10 \%)$ y genitales $(<1 \%)^{2}$.

En países en desarrollo, el linfedema secundario se debe principalmente a infecciones que comprometen el sistema linfático (filariasis), mientras que en países desarrollados se relaciona más frecuen-
Departamento de Cirugía Plástica, Clínica Las Condes. Santiago, Chile.

2Departamento de Cirugía Plástica y Quemados, Hospital del Trabajador. Santiago, Chile.

${ }^{3}$ Lymphedema International Center. Hiroshima University Hospital. Hiroshima, Japan.

Recibido el 9 de octubre de 2017, aceptado para publicación el 18 de diciembre de 2017.

Correspondencia a: Dr. Nicolás Pereira C. npereira@clc.cl 
Figura 1. Edema con fóvea en la extremidad inferior de un paciente con linfedema secundario. El edema corresponde a la acumulación de fluido linfático en el intersticio.

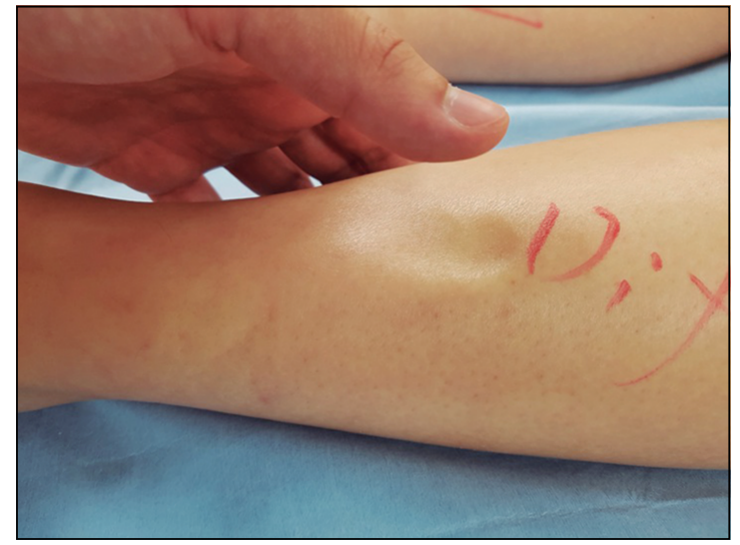

temente al tratamiento quirúrgico del cáncer. La incidencia luego de una disección axilar por cáncer de mama es $29-49 \%$ y en una biopsia de linfonodo centinela $5-7 \% \%^{3}$. Puede ocurrir como consecuencia del tratamiento de otros tipos de cáncer como melanoma $16 \%$, ginecológico $20 \%$, genitourinario $10 \%$, cabeza y cuello $4 \%$, sarcomas $30 \%{ }^{4}$.

Si bien no hay cura para el linfedema, el manejo debe estar a cargo de un equipo multidisciplinario. Uno de los tratamientos más comunes es la terapia descongestiva compleja (TDC) que incluye el uso de prendas de compresión. El tratamiento quirúrgico es una alternativa cuando las medidas conservadoras ya no son suficientes. A continuación se presenta una actualización en el diagnóstico y tratamiento del linfedema de extremidades.

Figura 2. Linfografía con ICG en paciente con linfedema de extremidades inferiores. Izquierda: En la pierna derecha se observa patrón lineal (normal) (flechas verdes), mientras que en la pierna izquierda se observa un patrón difuso de dermal backflow (flecha roja). Derecha: Figura esquemática de los hallazgos de la linfografía con ICG.

\section{Diagnóstico y etapificación}

\section{Diagnóstico}

Las causas de linfedema primario o secundario pueden ser identificadas a través de la historia clínica. En el examen físico, la medición volumétrica de la extremidad es lo principal para diagnóstico y el seguimiento de la progresión. Si bien, existen numerosas formas disponibles para medir el linfedema (desplazamiento de agua, mediciones circunferenciales, perímetro y espectrometría de bioimpedancia), no hay un método universalmente aceptado. Una vez hecho el diagnóstico clínico, se deben realizar estudios para determinar la extensión de la enfermedad y el status funcional del sistema linfático.

La linfocintigrafía isotópica ha sido considerada por mucho tiempo gold standard para el diagnóstico de linfedema ${ }^{5}$. Se realiza una inyección subdérmica de contraste no ionizante (Tc 99m, Tc 99m-HAS, o Tc 99m dextrán) en los espacios interdigitales que se sigue con gamma cámara. Entrega una evaluación estática y dinámica del flujo linfático, evidenciando zonas de bloqueo y/o flujo reverso. El hallazgo linfocintigráfico de vasos linfáticos principales sin colaterales permite predecir una buena respuesta al tratamiento conservador con terapia descongestiva compleja en etapas iniciales ${ }^{6}$.

La linfo-resonancia magnética (MRL) es capaz de mostrar cambios anatómicos detallados en los vasos linfáticos y linfonodos. En el linfedema primario, hay tres tipos principales de malformaciones del sistema linfático que se pueden representar: I) Sólo linfonodos afectados; II) sólo vasos linfáticos afectados, y III) tanto vasos linfáticos como linfonodos afectados. En linfedema secundario, MRL es capaz de demostrar claramente vasos linfáticos colectores tortuoso y dilatado en extremidades linfoedematosas. Proporciona información sobre el status funcional del transporte de linfa en los vasos linfáticos y en linfonodos mediante la visualización en tiempo real del flujo linfático contrastado en los canales linfáticos y dentro de los linfonodos ${ }^{7}$.

En la linfografía con verde indocianina (ICG) se realiza una inyección subdérmica de ICG en los espacios interdigitales de la extremidad y se observa mediante una cámara de fluorescencia cercana al infrarrojo (ej. SPY ${ }^{\circledR}$, PDE-neo®). Permite evaluar el sistema linfático en tiempo real, identificando la presencia y ubicación de vasos linfáticos permeables. Es preciso para estadificar el linfedema según su funcionalidad en patrón lineal (normal) y patrón de dermal backflow (anormal: splash, stardust, difuso) $)^{8,9}$ (Figura 2), así como orientar en la indicación del manejo quirúrgico. 


\section{Etapificación}

Se han propuesto múltiples esquemas de etapificación del linfedema. Probablemente, el más utilizado es el de la Sociedad Internacional de Linfología, el cual lo divide en 4 etapas $^{10}$. En la Tabla 1 se detalla la etapificación.

En cuanto a la severidad, es leve cuando el exceso de volumen de la extremidad afectada es $<20 \%$, moderado $20-40 \%$ y severo $>40 \%$.

\section{Tratamiento}

Los objetivos principales en el manejo del linfedema son limitar la morbilidad del paciente, mejorar la funcionalidad y la calidad de vida. Con el fin de lograr esto, se debe disminuir el edema, mejorar la higiene, reducir las tasas de infección, mejorar la movilidad de los pacientes y disminuir la carga de las terapias diarias. Por lo tanto, estos factores se deben tener en cuenta al momento de evaluar los resultados de cualquier tratamiento del linfedema.

Existen procedimientos que buscan prevenir el desarrollo del linfedema. Una vez establecido, el tratamiento puede ser conservador y quirúrgico. El tratamiento quirúrgico, a su vez, incluye procedimientos fisiológicos (reconstructivos) y resectivos.

\section{Tratamiento preventivo}

Existen estrategias de prevención del linfedema secundario al tratamiento oncológico, tanto para la extremidad superior como inferior.

La técnica de mapeo axilar reverso diferencia los linfáticos que drenan la mama de los que drenan el brazo para evitar dañarlos y en caso de seccionarlos, realizar anastomosis linfático-linfáticas y disminuir el riesgo de linfedema ${ }^{11}$. En un estudio prospectivo con 20 meses de seguimiento, pacientes con disección axilar presentaron linfedema extremidad superior secundario a cáncer de mama en un $33 \%$ mientras que en las pacientes que se agregaba el mapeo axilar reverso fue del $6 \%{ }^{12}$.

El abordaje linfático microquirúrgico preventivo curativo ( $L Y M P H A$, por sus siglas en inglés) es una técnica quirúrgica de prevención primaria de linfedema luego de la disección axilar ${ }^{13}$ o ilioinguinal ${ }^{14}$, como parte del tratamiento oncológico. Consiste en la realización de anastomosis linfático-venosas múltiples al momento de la linfadenectomía, entre los linfáticos principales seccionados que drenan la extremidad superior o inferior y ramas de la vena axilar o safena, respectivamente.

En el caso de linfedema de extremidad inferior
Tabla 1. Etapificación del linfedema según la Sociedad Internacional de Linfología ${ }^{10}$

\begin{tabular}{|ll|}
\hline Etapa & Características \\
0 & $\begin{array}{l}\text { Condición subclínica o latente. No hay edema evidente, pero el } \\
\text { transporte linfático está alterado. Pueden pasar meses o años antes } \\
\text { que se haga evidente el edema }\end{array}$ \\
I & $\begin{array}{l}\text { Acumulación precoz de fluido proteináceo reversible con elevación } \\
\text { de la extremidad o terapia compresiva. Puede ocurrir edema con } \\
\text { fóvea }\end{array}$ \\
II & $\begin{array}{l}\text { La elevación/compresión por sí sola ya no resuelve el edema y existe } \\
\text { fóvea }\end{array}$ \\
Temprano & $\begin{array}{l}\text { El edema con o sin fóvea, existe desarrollo de fibrosis en los tejidos } \\
\text { Elefantiasis linfostática. El tejido es firme (fibrótico) y hay ausencia } \\
\text { de fóvea. Se desarrollan acantosis, depósitos de grasa, crecimiento } \\
\text { verrucoso y otros cambios tróficos de la piel }\end{array}$ \\
\hline
\end{tabular}

secundario a la linfadenectomía pelviana por tratamiento oncológico, la prevención del linfedema de la extremidad contralateral se puede lograr realizando múltiples anastomosis linfático-venosas $(\mathrm{LVA})^{15} \mathrm{o}$ anastomosis linfático-venosas de los vasos eferentes de linfonodos inguinales ${ }^{16}$ antes que aparezcan los síntomas. Esto se indica cuando existe disfunción linfática diagnosticada con linfografía ICG en la extremidad sana (linfedema subclínico).

\section{Tratamiento conservador}

El tratamiento conservador ha sido un pilar fundamental y es la forma de iniciar el tratamiento del linfedema. La terapia descongestiva compleja (TDC) debe ser realizada por un profesional especializado en linfedema. Tiene una fase inicial reductiva (3-8 semanas) que consiste en el drenaje linfático manual, vendaje multicapa, ejercicios terapéuticos, cuidado de la piel, educación y autocuidado, compresión elástica; y una fase de mantenimiento que contempla el autodrenaje linfático permanente, ejercicios, cuidado de la piel y uso de prendas/vendaje de compresión ${ }^{10}$.

La TDC, logra un 40-60\% de reducción del exceso de volumen en pacientes con edema y fóvea ${ }^{17}$. Estas terapias tienen sus detractores debido a su carácter indefinido y a la amplia variabilidad en la calidad de los tratamientos y prendas compresivas.

Las medidas preventivas para el linfedema con evidencia científica son mantener el peso normal, evitar la ganancia de peso $^{18}$ y participar en programas de ejercicios supervisados ${ }^{19}$. 


\section{Tratamiento quirúrgico}

Procedimientos fisiológicos o reconstructivos

Actualmente, los procedimientos fisiológicos más comúnmente realizados son las anastomosis linfático-venosas y la transferencia de linfonodos vascularizados.

\section{- Anastomosis linfático-venosas (LVA)}

Descrita en 1969, esta técnica tiene como objetivo redirigir el exceso de fluido linfático a la circulación venosa y así superar zonas de obstrucción ${ }^{20}$. Los avances en los microscopios, el desarrollo de instrumental súperfino y atraumático, y el perfeccionamiento de la imagenología utilizada, han mejorado nuestra capacidad de realizar esta técnica.

No hay consenso respecto al momento de la cirugía, el número, localización y configuración de las LVA. Existe una relación directa entre el número de LVA y la reducción del volumen ${ }^{21}$, sin embargo, hay evidencia que sugiere que basta con una LVA funcionante para obtener resultados satisfactorios ${ }^{22}$.

La técnica comienza con la identificación de vasos linfáticos funcionales con ICG, los cuales se marcan en la piel. Se realiza una incisión transversal para identificar y aislar un vaso linfático y su vénula adyacente. Se realiza un bypass (LVA) utilizando nylon 11-0 y 12-0 con aguja 50-65 um términoterminal o latero-término. Una LVA es más simple y efectiva mientras más precoz se realice ${ }^{23}$.

El éxito de una LVA consiste en identificar un vaso linfático funcional y una vena apropiada compatible en tamaño, localización y que presente mínimo reflujo al seccionarla. Koshima et al resuelven este problema utilizando técnicas de supermicrocirugía para anastomosar un vaso linfático subdérmico con una vena adyacente menores a $0,8 \mathrm{~mm}^{24}$ (Figura 3).

Figura 3. Anastomosis linfático-venosa con técnica de supermicrocirugía (vasos de 0,35 mm). Se observan los capilares nutricios del vaso linfático. Asterisco: vena; flecha: vaso linfático.
Diversos estudios han demostrado la eficacia de la LVA en el tratamiento del linfedema. Muchos de estos reportes incluyen un limitado número de pacientes, pero demuestran una reducción aproximada del 35 al 50\% de la circunferencia o el volumen, en seguimientos mayores a 1 año $^{25}$. En un seguimiento a largo plazo de 90 pacientes que fueron sometidos a LVA, se encontró una mejoría objetiva en el $42 \%$, mejoría subjetiva en el $73 \%$ y una reducción media del volumen de un $44 \%$ en todos los pacientes ${ }^{26}$. Chang et al reportó un análisis prospectivo de LVA en 100 pacientes consecutivos con 12 meses de seguimiento, encontrando una reducción media del volumen del $61 \%$ en las primeras etapas del linfedema en la extremidad superior y el $17 \%$ en estadio avanzado de linfedema ${ }^{23}$. Cabe destacar que las mejorías más importantes se observan en la etapa temprana del linfedema de extremidad superior, con una meseta después de 1 año. Otros estudios han corroborado estos hallazgos logrando, además, una disminución de las tasas de infección y la suspensión de las prendas de compresión después de $\mathrm{LVA}^{25}$. Las complicaciones de la LVA son inusuales y mínima, incluyendo infección, fístula linfática y problemas de cicatrización de la herida. Los beneficios de la técnica son lo mínimamente invasivo, escaso dolor y posibilidad de alta a las 24-48 horas.

\section{- Transferencia de linfonodos vascularizados (VLNT)}

Descrito inicialmente por Becker et al para el tratamiento del linfedema ${ }^{27}$, es un procedimiento que consiste en la transferencia de un colgajo libre de linfonodos a la extremidad afectada mediante microcirugía.

Los mecanismos que explican su funcionamiento son que actuarían como una "esponja o bomba" que atrae linfa a los linfonodos y pasa al sistema venoso mediante conexiones linfático-venosas naturales dentro del colgajo ${ }^{28}$, o a través de linfoangiogénesis. En teoría, los VLNT producen mediadores linfoangiogénicos que actúan localmente para estimular el crecimiento e inosculación de vasos linfáticos a la red linfática de $\mathrm{VLNT}^{29}$. El principal mediador linfoangiogénico propuesto es el factor de crecimiento vascular endotelial C (VEGF-C).

Los linfonodos más utilizados para VLNT son los inguinales, supraclaviculares, submentales, torácicos y gastroepiploicos ${ }^{3,30}$.

Los linfonodos inguinales superficiales son los más frecuentemente utilizados y se pueden elevar en un colgajo inguinal basado en la arteria perforante de la circunfleja ilíaca superficial (SCIP $)^{31}$, así como en continuidad con un colgajo abdominal 
basado en la arteria perforante de epigástrica inferior profunda (DIEP) o en la arteria epigástrica inferior superficial (SIEA) para reconstrucción mamaria ${ }^{32}$. Dentro de sus ventajas se menciona una cicatriz de zona dadora oculta, anatomía confiable y la presencia de múltiples linfonodos. Las desventajas son el pequeño calibre de los vasos y lo corto del pedículo (SCIP), y el potencial de linfedema iatrogénico de la extremidad inferior como secuela. Para prevenir esta complicación, diversos estudios demuestran que los linfonodos que drenan la extremidad inferior se localizan medial a la arteria femoral, bajo el pliegue inguinal (en el triángulo femoral), mientras que los que drenan el abdomen inferior se ubican lateral, más superficial a la arteria femoral, entre el ligamento y el pliegue inguinal ${ }^{31}$. El "mapeo linfático reverso" permite diferenciar los linfonodos que drenan la extremidad inferior con un radionucleótido marcado, de los linfonodos que drenan el abdomen inferior con $\mathrm{ICG}^{33}$. Aunque el riesgo de linfedema iatrogénico de la zona dadora luego de VLNT es bajo, es un riesgo real y debe ser discutido con el paciente.

El colgajo supraclavicular se basa en los vasos cervicales transversos. Generalmente se eleva sin isla cutánea y deja una zona dadora discreta. Posee menos linfonodos que el inguinal y submental ${ }^{31}$. Se debe tener cuidado al elevarlo con los nervios supraclaviculares, y en el lado izquierdo, por riesgo de dañar el conducto torácico.

El colgajo submental se basa en la arteria submental e incluye hasta 6 linfonodos cervicales del nivel $1 \mathrm{~A}$ y $1 \mathrm{~B}^{31}$. Las ventajas son el número de linfonodos, anatomía confiable, adecuado calibre de la arteria submental y facial, facilidad de elevación, colgajo delgado y potencial limitado de linfedema iatrogénico. Las desventajas incluyen el riesgo de dañar el nervio marginal mandibular, parálisis del platisma y la cicatriz evidente en el cuello.

Los linfonodos torácicos se basan en la arteria torácica lateral o ramas de la arteria toracodorsal e incluyen el nivel I de los linfonodos axilares. Tiene el riesgo de provocar linfedema iatrogénico en la extremidad superior. Se puede utilizar el "mapeo linfático reverso" para identificar los linfonodos adecuados para la transferencia y evitar esta complicación $^{33}$.

El colgajo de linfonodos gastroepiploicos se basa en los vasos del mismo nombre y puede ser obtenido por vía laparoscópica ${ }^{34}$. Tiene la ventaja que puede dividirse en dos colgajos independientes para diferentes zonas receptoras.

La selección de la zona receptora se basa en la localización del linfedema (extremidad superior o inferior), disponibilidad de vasos receptores, cicatrices previas, apariencia estética y la experiencia del cirujano. Cuando se propone la axila o ingle luego de una cirugía previa en la zona, se debe remover todo el tejido cicatricial hasta plano vital y reemplazarlo por un colgajo bien vascularizado. Además, los colgajos en esta ubicación se pueden ocultar de mejor manera y otorgar un mejor resultado estético. Por el contrario, las zonas distales como receptora de VLNT son más fáciles de disecar y aportarían el efecto "esponja" como mecanismo de drenaje, por lo que en teoría comenzarían a funcionar inmediatamente.

Diversos estudios han reportado reducciones significativas en la circunferencia o el volumen de $30 \%$ a $60 \%$ luego de $\mathrm{VLNT}^{35}$. En la mayoría de los casos se trata de estudios retrospectivos, sin embargo, Cheng et al, en un trabajo prospectivo, encontraron una mayor reducción significativa en la circunferencia de 10 pacientes que recibieron VLNT (40\%) comparado con 10 pacientes controles que eligieron tratamiento conservador $(8 \%)$ en un período de seguimiento de 39 meses $^{30}$. Patel et al reportaron un $24 \%$ de reducción en la circunferencia de la extremidad superior y un $35 \%$ en la extremidad inferior, en un estudio prospectivo de 25 pacientes con seguimiento a 12 meses $^{36}$.

\section{Procedimientos resectivos}

Descrito inicialmente por Charles en 1901 para el tratamiento excisional del linfedema escrotal ${ }^{37}$, en extremidades consiste en escisión radical circunferencial del tejido linfoedematoso hasta la fascia profunda y cobertura inmediata con injerto dermoepidérmico de la piel resecada. Debido a lo desfigurante del procedimiento, está indicado solamente para los casos más severos. El procedimiento de Charles modificado utiliza terapia con presión negativa después de la reducción inicial, realizando el injerto a los 5 a 7 días.

La reducción radical del linfedema con preservación de perforantes (RRPP) se basa en un mejor conocimiento de la irrigación de la piel, permitiendo una resección extensa manteniendo la perfusión. Esta técnica ha mostrado resultados considerables, siendo particularmente útil en linfedema de extremidades inferiores con una reducción del $52 \%$, más que para extremidades superiores ${ }^{38}$.

La liposucción puede ser utilizada de manera aislada o en combinación a otros procedimientos para tratar el linfedema. Remueve el tejido graso infiltrado que no responde a TCD o a procedimientos fisiológicos, pero lamentablemente no elimina la necesidad de tratamiento compresivo continuo en 
Figura 4. Algoritmo general para el tratamiento del linfedema. ICG: verde indocianina; MRL: linfo-resonancia magnética; LVA: anastomosis linfático-venosas; VLNT: transferencia de linfonodos vascularizados.

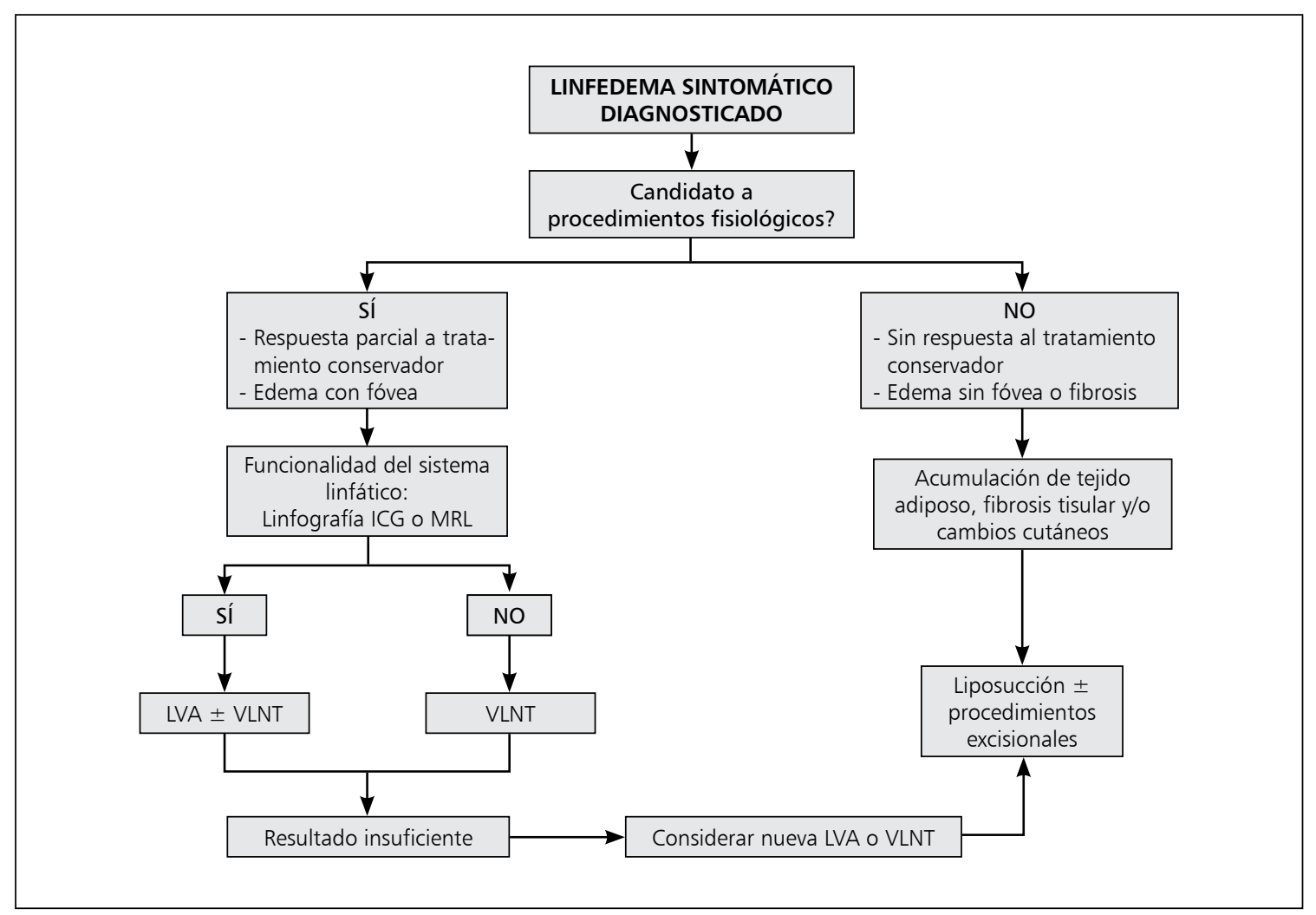

el posoperatorio ${ }^{39}$. A pesar de estas deficiencias, la liposucción ha demostrado disminuir el edema, las tasas de infección en la extremidad linfoedematosa, por lo que continúa siendo una herramienta muy útil para el tratamiento del linfedema severo y como técnica complementaria ${ }^{39}$.

\section{Enfrentamiento terapéutico del paciente con linfedema}

Se debe realizar una evaluación completa de manera de poder ofrecer un tratamiento individualiza$\mathrm{do}^{32,40}$ (Figura 4). El tratamiento se basa en la etapa clínica, consideraciones anatómicas y el deseo del paciente. Los casos que presentan edema con fóvea y respuesta parcial al tratamiento conservador, son candidatos para procedimientos fisiológicos ya que el edema se debe principalmente a la acumulación de fluido linfático que puede ser resuelto mediante LVA o VLNT. En casos más avanzados, el aumento de volumen se debe a la acumulación de tejido adiposo, por lo que son candidatos a procedimientos resectivos.

Para decidir qué procedimiento fisiológico realizar, se debe hacer el diagnóstico con linfocintigrafía y evaluar la funcionalidad del sistema linfático mediante MRL o linfografía con ICG. Si el sistema linfático aún es funcional el paciente es candidato para LVA. Si no se logran identificar vasos linfáticos, se ofrece la realización de VLNT, más aún si tiene antecedentes de linfadenectomía. Existen casos en que LVA y VLNT se pueden realizar en la misma cirugía.

Cuando se realiza una VLNT, el lecho receptor se debe preparar resecando todo el tejido cicatricial como resultado de la cirugía previa y/o radioterapia, liberando la compresión venosa y permitiendo la linfangiogénesis entre el colgajo y el lecho receptor. En los casos de linfedema asociado a cáncer de mama, se puede realizar la reconstrucción mamaria simultáneamente a la VLNT ${ }^{32}$. En los casos con exceso de adiposidad subcutánea residual, se pueden realizar procedimientos resectivos 6-12 meses después de la primera cirugía.

Durante el período posoperatorio, los pacientes deben discontinuar la terapia física y suspender el uso de prendas elásticas por 2-4 semanas y reasumir progresivamente sus actividades normales según tolerancia. Luego del período posoperatorio inicial, 


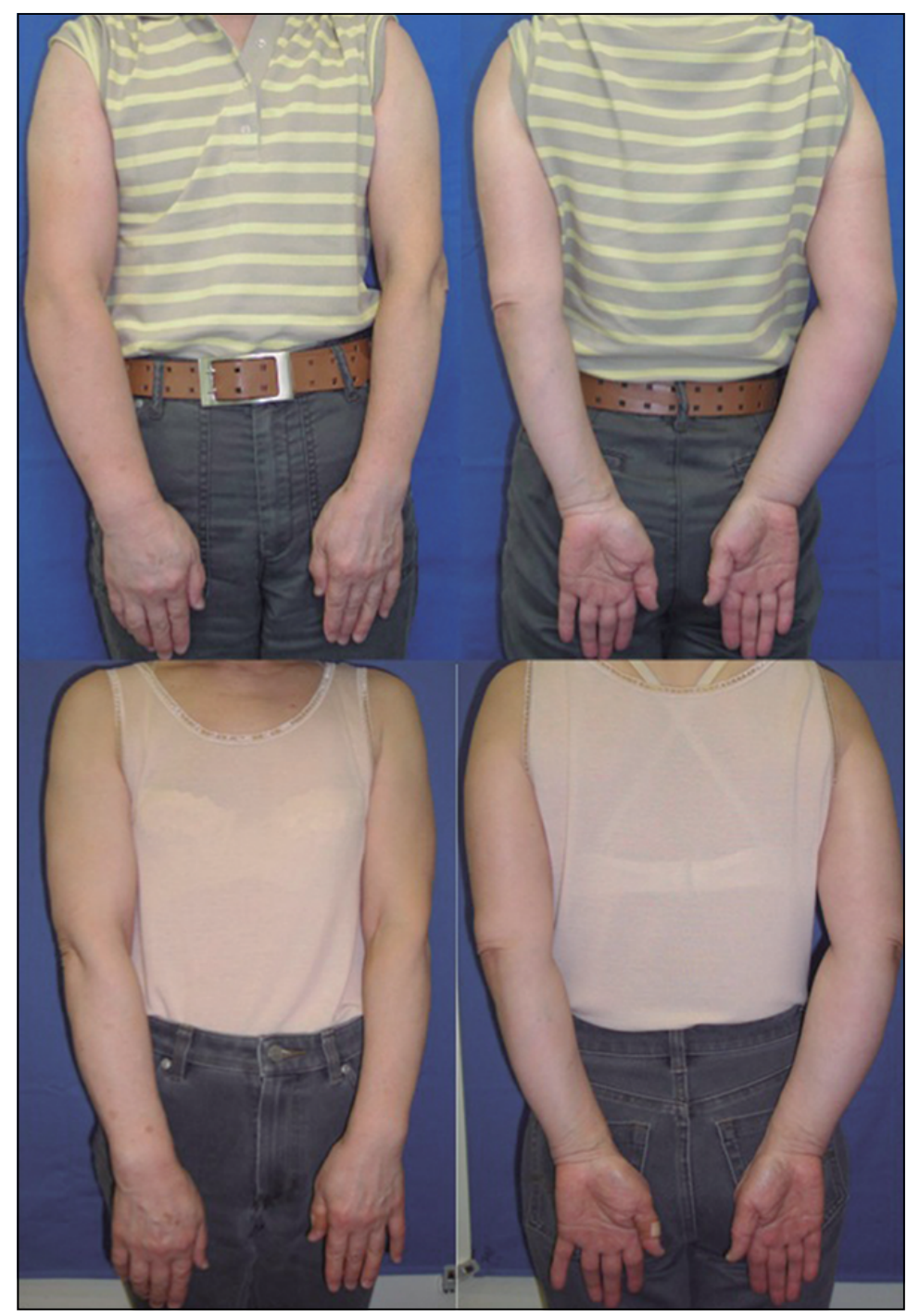

Figura 5. Paciente de 61 años sexo femenino con linfedema de extremidad superior derecha secundario al tratamiento de un cáncer de mama. Arriba: antes del tratamiento con anastomosis linfático-venosas; Abajo: a los 13 meses posoperatorio.

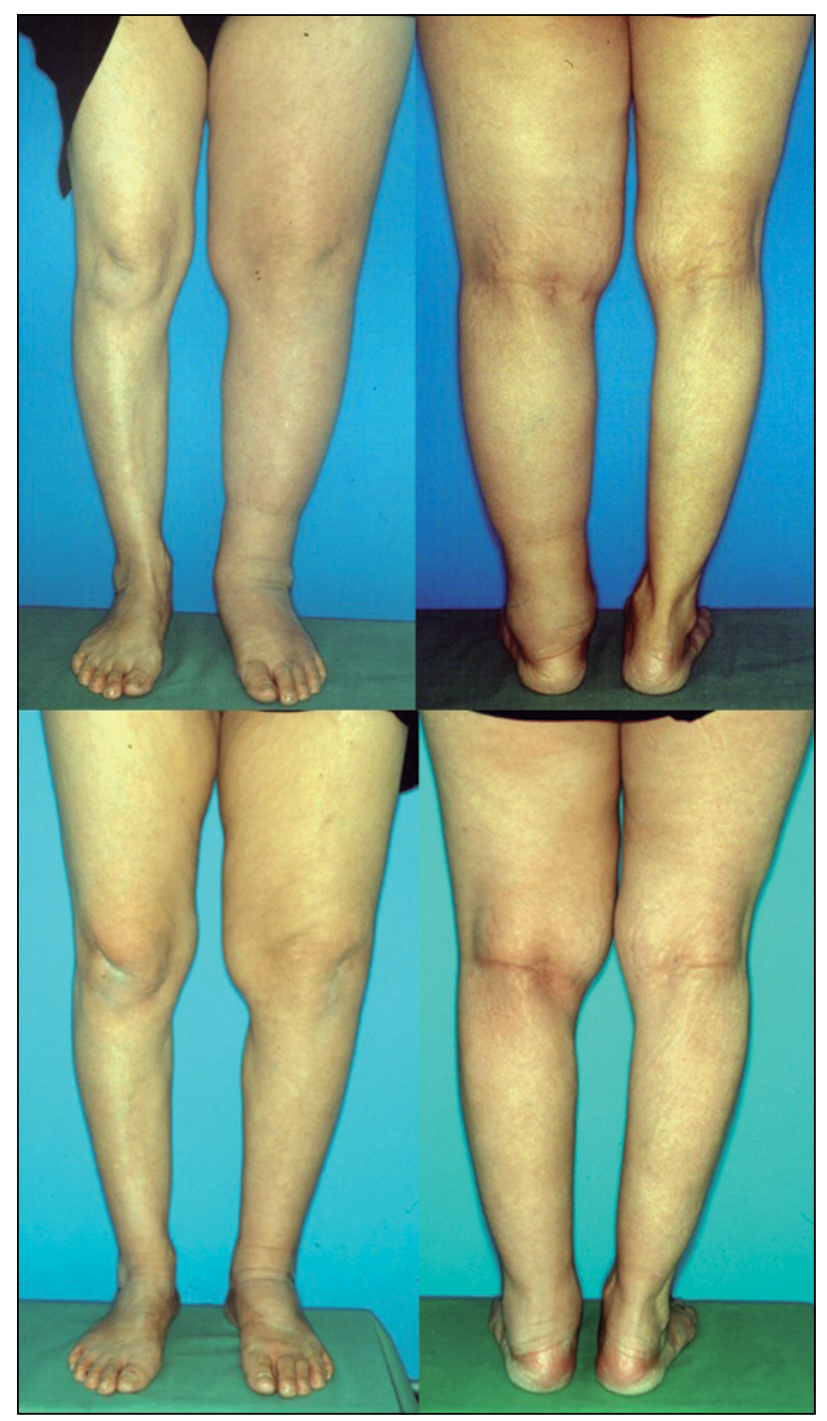

Figura 6. Paciente de 49 años sexo femenino con linfedema de extremidad inferior izquierda secundario al tratamiento de un cáncer ginecológico. Arriba: antes del tratamiento con anastomosis linfático-venosas; Abajo: a los 17 meses posoperatorio. los pacientes deben controlarse periódicamente. Los resultados se evidencian desde los 3 meses y se estabilizan al año posoperatorio. Los resultados se evalúan de acuerdo a la mejoría subjetiva del paciente, medidas perimetrales y volumétricas, frecuencia de episodios de celulitis y cuestionarios de calidad de vida (Figura 5 y Figura 6).

\section{Conclusiones}

El tratamiento del linfedema es desafiante, está en continuo desarrollo y debe ser realizado por un equipo multidisciplinario. Las nuevas estrategias diagnósticas y las emergentes técnicas microquirúrgicas tienen el potencial de reconstruir fisiológicamente áreas de drenaje linfático deteriorado. El éxito depende de una buena elección de los pacientes y la realización de un tratamiento individualizado. Si bien aún estamos lejos de ganar la batalla contra el linfedema; la comprensión del proceso fisiopatológico, el entendimiento del diagnóstico adecuado, la investigación y el desarrollo de estudios prospectivos nos ayudarán a mejorar la calidad de vida de nuestros pacientes. 


\section{Responsabilidades éticas}

Protección de personas y animales. Los autores declaran que para esta investigación no se han realizado experimentos en seres humanos ni en animales.

Confidencialidad de los datos. Los autores declaran que en este artículo no aparecen datos de pacientes.

Derecho a la privacidad y consentimiento informado. Los autores declaran que en este artículo no aparecen datos de pacientes.

Conflicto de intereses: No hay.

\section{Referencias}

1. Basta MN, Gao LL, Wu LC. Operative treatment of peripheral lymphedema: a systematic meta-analysis of the efficacy and safety of lymphovenous microsurgery and tissue transplantation. Plast Reconstr Surg. 2014;133:905-13.

2. Raskin ER, Slavin SA, Borud LJ. Lymphedema. En Guyuron B, Eriksson E, Persing JA, editores. Plastic Surgery: Indications and Practice. 1ra ed. Philadelphia: Saunders Elsevier; 2009. p. 195-8.

3. DiSipio T, Rye S, Newman B, Hayes S. Incidence of unilateral arm lymphoedema after breast cancer: A systematic review and meta-analysis. Lancet Oncol. 2013;14:500-15.

4. Cormier JN, Askew RL, Mungovan KS, Xing Y, Ross MI, Armer JM. Lymphedema beyond breast cancer: a systematic review and meta-analysis of cancer-related secondary lymphedema. Cancer 2010;116:5138-49.

5. Maegawa J, Mikami T, Yamamoto Y, Satake T, Kobayashi S. Types of lymphoscintigraphy and indications for lymphaticovenous anastomosis. Microsurgery 2010;30:437-42.

6. Hwang JH, Choi JY, Lee JY, Hyun SH, Choi Y, Choe YS, et al. Lymphscintigraphy predicts response to complex physical therapy in patients with early stage extremity lymphedema. Lymphology 2007;40:172-6.

7. Arrivé L, Derhy S, El Mouhadi S, Monnier-Cholley L, Menu Y, Becker C. Noncontrast magnetic resonance lymphography. J Reconstr Microsurg. 2016;32:80-6.

8. Yamamoto T, Yamamoto N, Doi K, Oshima A, Yoshimatsu H, Todokoro $\mathrm{T}$, et al. Indocyanine green-enhanced lymphography for upper extremity lymphedema: a novel severity staging system using dermal backflow patterns. Plast Reconstr Surg. 2011;128:941-7.

9. Yamamoto T, Matsuda N, Doi K, Oshima A, Yoshimatsu H, Todokoro T, et al. The earliest finding of indocyanine green lymphography in asymptomatic limbs of lower extremity lymphedema patients secondary to cancer treatment: The modified dermal backflow stage and concept of subclinical lymphedema. Plast Reconstr Surg. 2011;128:314e-21e.

10. International Society of Lymphology. The diagnosis and treatment of peripheral lymphedema: 2013 Consensus Document of the International Society of Lymphology. Lymphology. 2013;46:1-11.

11. Ahmed M, Rubio IT, Kovacs T, Klimberg VS, Douek M. Systematic review of axillary reverse mapping in breast cancer. Br J Surg. 2016;103:170-8.

12. Yue T, Zhuang D, Zhou P, Zheng L, Fan $\mathrm{Z}$, Zhu J, et al. prospective study to assess the feasibility of axillary reverse mapping and evaluate its effect on preventing lymphedema in breast cancer patients. Clin Breast Cancer 2015;15:301-6.

13. Boccardo F, Casabona F, De Cian F, Friedman D, Villa G, Bogliolo S, et al. Lymphedema microsurgical preventive healing approach: a new technique for primary prevention of arm lymphedema after mastectomy. Ann Surg Oncol. 2009;16:703-8.

14. Boccardo F, Valenzano M, Costantini S, Casabona F, Morotti M, Sala P, et al. LYMPHA Technique to Prevent Secondary Lower Limb Lymphedema. Ann Surg Oncol. 2016;23:3558-63.

15. Onoda S, Todokoro T, Hara H, Azuma $\mathrm{S}$, Goto A. Minimally invasive multiple lymphaticovenular anastomosis at the ankle for the prevention of lower leg lymphedema. Microsurgery 2014;34:3726.

16. Yamamoto $\mathrm{T}$, Yamamoto $\mathrm{N}$, Yamashita M, Furuya M, Hayashi A, Koshima I.
Efferent Lymphatic Vessel Anastomosis: Supermicrosurgical Efferent Lymphatic Vessel-to-Venous Anastomosis for the Prophylactic Treatment of Subclinical Lymphedema. Ann Plast Surg. 2016;76:424-7.

17. Warren AG, Brorson H, Borud LJ, Slavin SA. Lymphedema: a comprehensive review. Ann Plast Surg. 2007;59:464-72.

18. Shaw C, Mortimer P, Judd PA. A Randomized Controlled Trial of Weight Reduction as a Treatment for Breast Cancer-related Lymphedema. Cancer 2007;110:1868-74.

19. McKenzie DC, Kalda AL. Effect of Upper Extremity Exercise on Secondary Lymphedema in Breast Cancer Patients: A Pilot Study. J Clin Oncol. 2003;21:463-6.

20. Yamada Y. The studies on lymphatic venous anastomosis in lymphedema. Nagoya J Med Sci. 1969;32:1-21.

21. Mihara M, Hara H, Tange S, Zhou HP, Kawahara M, Shimizu Y, et al. Multisite Lymphaticovenular Bypass Using Supermicrosurgery Technique for Lymphedema Management in Lower Lymphedema Cases. Plast Reconstr Surg. 2016;138:262-72.

22. Seki Y, Yamamoto T, Yoshimatsu H, Hayashi A, Kurazono A, Mori M, et al. The Superior-Edge-of-the-Knee Incision Method in Lymphaticovenular Anastomosis for Lower Extremity Lymphedema. Plast Reconstr Surg. 2015; 136:665e-75e.

23. Chang DW, Suami H, Skoracki R. A prospective analysis of 100 consecutive lymphovenous bypass cases for treatment of extremity lymphedema. Plast Reconstr Surg. 2013;132:1305-14.

24. Koshima I, Yamamoto T, Narushima M, Mihara M, Lida T. Perforator flaps and supermicrosurgery. Clin Plast Surg. 201;37:683-9.

25. Campisi C, Bellini C, Campisi C, Accogli S, Bonioli E, Boccardo F. Microsurgery 
for lymphedema: Clinical research and long-term results. Microsurgery 2010;30:256-60.

26. O’Brien BM, Mellow CG, Khazanchi RK, Dvir E, Kumar V, Pederson WC. Longterm results after microlymphaticovenous anastomoses for the treatment of obstructive lymphedema. Plast Reconstr Surg. 1990;85:562-72.

27. Becker C, Assouad J, Riquet M, Hidden G. Postmastectomy lymphedema: longterm results following microsurgical lymph node transplantation. Ann Surg. 2006;243:313-5.

28. Cheng MH, Huang JJ, Wu CW, Yang CY, Lin CY, Henry SL, et al. The mechanism of vascularized lymph node transfer for lymphedema: Natural lymphaticovenous drainage. Plast Reconstr Surg. 2014;133:192e-198e

29. Aschen SZ, Farias-Eisner G, Cuzzone DA, Albano NJ, Ghanta S, Weitman ES, et al. Lymph node transplantation results in spontaneous lymphatic reconnection and restoration of lymphatic flow. Plast Reconstr Surg. 2014;133:301-10.

30. Cheng MH, Chen SC, Henry SL, Tan
BK, Lin MC, Huang JJ. Vascularized groin lymph node flap transfer for postmastectomy upper limb lymphedema: Flap anatomy, recipient sites, and outcomes. Plast Reconstr Surg. 2013;131:1286-98.

31. Patel KM, Chu SY, Huang JJ, Wu $\mathrm{CW}$, Lin CY, Cheng MH. Preplanning vascularized lymph node transfer with duplex ultrasonography: An evaluation of 3 donor sites. Plast Reconstr Surg Glob Open. 2014;2:e193.

32. Masià J, Pons G, Rodríguez-Bauzà E. Barcelona Lymphedema Algorithm for Surgical Treatment in Breast CancerRelated Lymphedema. J Reconstr Microsurg. 2016;32:329-35.

33. Dayan JH, Dayan E, Smith ML. Reverse lymphatic mapping: A new technique for maximizing safety in vascularized lymph node transfer. Plast Reconstr Surg. 2015;135:277-85.

34. Johnston ME, Socas J, Hunter JL, Ceppa EP. Laparoscopic Gastroepiploic Lymphovascular Pedicle Harvesting for the Treatment of Extremity Lymphedema: A Novel Technique. Surg Laparosc
Endosc Percutan Tech. 2017;27:e40-e43.

35. Raju A, Chang DW. Vascularized lymph node transfer for treatment of lymphedema: A comprehensive literature review. Ann Surg. 2015;261:1013-23.

36. Patel KM, Lin CY, Cheng MH. A prospective evaluation of lymphedemaspecific quality-of-life outcomes following vascularized lymph node transfer. Ann Surg Oncol. 2015;22:2424-30.

37. Charles RH. The surgical technique and operative treatment of elephatiasis of the generative organs based on a series of 140 consecutive successful cases. Indian Medical Gazette. 1901;36:84-99.

38. Salgado CJ, Mardini S, Spanio S, Tang WR, Sassu P, Chen HC. Radical reduction of lymphedema with preservation of perforators. Ann Plast Surg. 2007;59:1739.

39. Brorson H. Liposuction in Lymphedema Treatment. J Reconstr Microsurg. 2016;32:56-65.

40. Kung TA, Champaneria MC, Maki JH, Neligan PC. Current Concepts in the Surgical Management of Lymphedema. Plast Reconstr Surg. 2017;139:1003e-13e. 